\title{
A bilateral Tauberian theorem
}

\author{
By Åke Pleijel
}

This note contains the proof of a bilateral Tauberian theorem which in incomplete form was used in my paper [2].

By the elementary proof the Tauberian theorem is reduced to two well-known theorems by Hardy and Littlewood, see [1].

\section{The Hardy and Littlewood theorems}

Let $\sigma$ be a real function of bounded variation on every finite subinterval of $0 \leqslant \lambda<+\infty$.

The following Abelian theorems are easily proved.

Theorem $A\{q\}$. If $0<q<1$ and $\sigma(\lambda)=o\left(\lambda^{q}\right)$ when $\lambda \rightarrow+\infty$, then

$$
\int_{0}^{\infty}(\lambda+t)^{-1} d \sigma(\lambda)=o\left(t^{q-1}\right), t \rightarrow+\infty
$$

Theorem $A\{0\}$. If $\sigma(\lambda)=\sigma(+\infty)+o(1)$ when $\lambda \rightarrow+\infty$, then

$$
\int_{0}^{\infty}(\lambda+t)^{-1} d \sigma(\lambda)=(\sigma(+\infty)-\sigma(0)) t^{-1}+o\left(t^{-1}\right), t \rightarrow+\infty .
$$

The conclusions are still valid if $t$ tends to infinity along half rays from the origin in the complex $t$-plane which are different from the real negative axis. To see this one has only to use the inequality $|\lambda+t| \geqslant(\lambda+|t|) \sin \frac{1}{2} \delta,|\arg t| \leqslant \pi-\delta$, when the necessary estimations are performed.

To formulate the corresponding Tauberian theorems we need

Definition 1. The function $\sigma$ belongs to $T^{s}$ if there is a real constant $C$ such that $d \sigma(\lambda)+C \lambda^{s-1} d \lambda$ is definite, $\geqslant 0$ or $\leqslant 0$, for sufficiently large values of $\lambda$.

The theorems are

Theorem $T\{q\}$. If $0<q<1, \sigma \in T^{q}$ and 
Åke PleiJel, $A$ bilateral Tauberian theorem

$$
\int_{0}^{\infty}(\lambda+t)^{-1} d \sigma(\lambda)=o\left(t^{q-1}\right), t \rightarrow+\infty
$$

then $\sigma(\lambda)=o\left(\lambda^{q}\right)$ when $\lambda \rightarrow+\infty$.

Theorem $T\{0\}$. If $\sigma \in T^{0}$ and

$$
\int_{0}^{\infty}(\lambda+t)^{-1} d \sigma(\lambda)=H t^{-1}+o\left(t^{-1}\right), t \rightarrow+\infty,
$$

then $\sigma(\lambda)=\sigma(+\infty)+o(1)$ when $\lambda \rightarrow+\infty$. The constant $H$ is related to $\sigma$ by $H=$ $\sigma(+\infty)-\sigma(0)$.

\section{Certain lemmas}

An addition of a constant to the function $\sigma$ of the previous section is evidently irrelevant. The same holds for the functions of this section since we are essentially only interested in their differentials. Thus the definition

$$
I^{k} \varphi(\lambda)=\int^{\lambda} \mu^{k} d \varphi(\mu)
$$

determines $I^{k} \varphi$ up to an additive constant. It is always possible to avoid divergence difficulties by taking the lower limit of integration positive. Obviously

$$
I^{j}\left(I^{k} \varphi\right)=I^{j+k} \varphi
$$

Definition 2. We write $\varphi(\lambda) \in \omega^{s}$ if $\varphi(\lambda)=o\left(\lambda^{s}\right)$ when $\lambda \rightarrow+\infty$ and $s>0$ or if $\varphi(\lambda)=\varphi(+\infty)+o\left(\lambda^{s}\right)$ when $\lambda \rightarrow+\infty$ and $s \leqslant 0$.

Consider $I^{k} \varphi$ when $\varphi \in \omega^{s}$. It is no restriction to assume $\varphi=o\left(\lambda^{s}\right)$ also when $s \leqslant 0$. From the relation

$$
I^{k} \varphi(\lambda)-I^{k} \varphi(A)=\frac{\varphi(\lambda)}{\lambda^{s}} \lambda^{k+s}-\frac{\varphi(A)}{A^{s}} A^{k+s}-k \int_{A}^{\lambda} \frac{\varphi(\mu)}{\mu^{s}} \mu^{k+s-1} d \mu
$$

it follows when $k+s>0$ that

$$
I^{k} \varphi(\lambda)=o\left(\lambda^{k+s}\right), \lambda \rightarrow+\infty
$$

If $k+s<0$ relation (1) shows the convergence of $I^{k} \varphi(\lambda)$ when $\lambda \rightarrow+\infty$. The transition to the limit $A \rightarrow+\infty$ then leads to a formula from which one concludes that

$$
I^{k} \varphi(\lambda)-I^{k} \varphi(+\infty)=o\left(\lambda^{k+s}\right), \lambda \rightarrow+\infty .
$$

Thus we have proved 
Lemma 1. If $\varphi \in \omega^{s}$ then $I^{k} \varphi \in \omega^{k+s}$ provided $k \neq-s$.

That the lemma is not valid without the condition $k \neq-s$ is seen by the example $\varphi(\lambda)=I^{s} \log \log \lambda$. This function belongs to $\omega^{s}$ when $s \neq 0$ but $I^{-s} \varphi(\lambda)=$ $\log \log \lambda$ is not in $\omega^{0}$.

Definition 3. We write $\varphi \in I^{s}$ if $I^{k} \varphi \in \omega^{k+s}$ for all $k \neq-s$.

Observe that it follows from lemma 1 that if $I^{k} \varphi \in \omega^{k+s}$ holds for an arbitrary value of $k$ it holds for all values $k \neq-s$. This leads to

Lemma 2. $\omega^{s}=I^{s}$ if $s \neq 0$ and $\omega^{0} \subset I^{0}$.

The function $\log \log \lambda$ belongs to $I^{0}$ but is not in $\omega^{0}$.

Lemma 3. $\varphi \in I^{s}$ implies $I^{k} \varphi \in I^{k+s}$.

Lemma 3 is an immediate consequence of the definition of $I^{s}$. The lemma is closely related to lemma 1 but is free from supplementary condition.

Lemma 4. The class $I^{s}$ is linear.

Lemma 5. If $\varphi(\lambda) \in I^{s}$ then $\varphi\left(\lambda^{k}\right) \in I^{k s}$.

Lemma 4 and 5 follow from the definition of $\omega^{s}$ and $I^{s}$.

Lemma 6. If $\varphi \in T^{s}$ then $I^{k} \varphi \in T^{k+s}$.

For if $d \varphi+C \lambda^{s-1} d \lambda$ is definite, the same is true for the differential obtained when $d \varphi+C \lambda^{s-1} d \lambda$ is multiplied by $\lambda^{k}$.

Lemma 7. If $\varphi_{1}, \varphi_{2} \in T^{s}$ then either $\varphi_{1}+\varphi_{2} \in T^{s}$ or $\varphi_{1}-\varphi_{2} \in T^{s}$.

If $d \varphi_{1}+C_{1} \lambda^{s-1} d \lambda \geqslant 0, d \varphi_{2}+C_{2} \lambda^{s-1} d \lambda \geqslant 0$ it follows by addition that $\varphi_{1}+\varphi_{2} \in T^{s}$. If $d \varphi_{1}+C_{1} \lambda^{s-1} d \lambda \geqslant 0, d \varphi_{2}+C_{2} \lambda^{s-1} d \lambda \leqslant 0$ subtraction yields $\varphi_{1}-\varphi_{2} \in T^{s}$.

Lemma 8. If $\varphi(\lambda) \in T^{s}$ then $\varphi\left(\lambda^{k}\right) \in T^{k s}$.

Lemma 9. If $s<r$ then $T^{s} \subset T^{r}, T^{s} \neq T^{r}$. Also $\omega^{s} \subset \omega^{r}, \omega^{s} \neq \omega^{r}$ and $I^{s} \subset I^{r}, I^{s} \neq I^{r}$.

Lemma 8 is obtained by a change of the independent variable and Lemma 9 is easily deduced from the definitions of $T^{s}, \omega^{s}$ and $I^{s}$.

\section{Unilateral theorems}

Let $\sigma$ be a function of the type in section 1 and assume that the integral of $\lambda^{-h} d \sigma(\lambda)$ converges absolutely when taken over a right side neighbourhood of the origin. Under this condition consider 
ÅKE PLEIJEL, $A$ bilateral Tauberian theorem

$$
\int_{0}^{\infty} \lambda^{-h}(\lambda+t)^{-1} d \sigma(\lambda)
$$

If this integral converges at infinity for one value $t=t_{\mathbf{0}}$, for instance for $t=0$, it is seen by introducing in (2)

$$
\beta(\lambda)=\int_{a}^{\lambda} \mu^{-n}\left(\mu+t_{0}\right)^{-1} d \sigma(\mu), \quad a>0,
$$

and by partial integration that (2) converges at infinity for every $t$. If

$$
\int_{0}^{\infty} \lambda(\lambda+t)^{-1} d I^{-h-1} \sigma(\lambda)
$$

is integrated by parts it follows that (2) is $o(1)$ when $t$ tends to infinity along half rays different from the negative real axis. Thus we have

Theorem 1. Necessary and sufficient for the convergence of (2) is $I^{-h-1} \sigma \in \omega^{0}$. The integral is then o(1) when $t \rightarrow \infty$ along half rays from the origin which are different from the negative real axis.

In the Abelian theorem for (2) which will be considered presently, it is assumed that $\sigma \in I^{s}$ when $s-h \neq$ integer or $I^{-s} \sigma \in \omega^{0}$ when $s-h=$ integer. These relations appear as conclusions in the corresponding Tauberian theorem. It follows that it is natural to suppose $s<h+1$. If $s=h+1$ the condition $I^{-s} \sigma \in \omega^{0}$ is equivalent to the existence of the integral (2) and also implies that this integral is $o(1)$ when $t \rightarrow \infty$. The Abelian and Tauberian theorems given below can therefore be extended in a trivial way to include also the case $s=h+1$ in which the Tauberian theorem holds without the Tauberian condition $\sigma \in T^{s}$. We restrict ourselves to the non-trivial case $s<h+1$.

The following Abelian and Tauberian theorems are proved in section 4 and 5 .

Theorem $A(0, \infty)$. If $s<h+1$ and $\sigma \in I^{s}$ or in case $s-h$ is an integer, if $I^{-s} \sigma \in \omega^{0}$, then

$$
\int_{0}^{\infty} \lambda^{-h}(\lambda+t)^{-1} d \sigma(\lambda)=t^{-1} p\left(t^{-1}\right)+o\left(t^{s-h-1}\right), \quad t \rightarrow+\infty,
$$

where $p$ is a polynomial.

Recall that if $s \neq 0$ the condition $\sigma \in I^{s}$ coincides with $\sigma \in \omega^{s}$ i.e. $\sigma(\lambda)=o\left(\lambda^{s}\right)$ when $s>0$ and $\sigma(\lambda)=\sigma(+\infty)+o\left(\lambda^{s}\right)$ when $s<0$. The condition $I^{-s} \sigma \in \omega^{0}$ is equivalent to the convergence at infinity of

$$
\int^{\infty} \lambda^{-s} d \sigma(\lambda)
$$

According to lemma 1 and definition 3 the relation $I^{-s} \sigma \in \omega^{0}$ implies that $\sigma \in I^{s}$ but the converse is not true. 
The conclusion of $A(0, \infty)$ also holds when $t$ tends to infinity along arbitrary half rays from the origin different from the negative real axis.

Theorem $T(0, \infty)$. If $s<h+1$, if $\sigma \in T^{s}$ and if, with a polynomial $p$,

$$
\int_{0}^{\infty} \lambda^{-h}(\lambda+t)^{-1} d \sigma(\lambda)=t^{-1} p\left(t^{-1}\right)+o\left(t^{s-h-1}\right), \quad t \rightarrow+\infty
$$

then $\sigma \in I^{s}$. If $s-h$ is an integer the result can be sharpened to $I^{-s} \sigma \in \omega^{0}$.

Remark. The assumption $\sigma \in T^{s}$ can be replaced by the slightly more general condition that $\sigma+\varphi \in T^{s}$ for a $\varphi$ satisfying $\varphi \in I^{s}$ or, in case $s-h=$ integer, $I^{-s} \varphi \in \omega^{0}$.

\section{Proof of the unilateral Abelian theorem}

Theorem $A(0, \infty)$ is easily reduced to $A\{q\}$ and $A\{0\}$. If $0<s-h<1$ the polynomial $t^{-1} p\left(t^{-1}\right)$ is irrelevant in $(3)$ and can be included in the remainder. When $s-h=0$ only the first term $a_{1} t^{-1}$ of $t^{-1} p\left(t^{-1}\right)$ is relevant. The left hand side of (3) can be written

$$
\int_{0}^{\infty}(\lambda+t)^{-1} d I^{-n} \sigma(\lambda)
$$

According to lemma 3 it follows from the condition $\sigma \in I^{s}$ of $A(0, \infty)$ that $I^{-h} \sigma \in I^{s-h}$ and if $s-h \neq 0$ we know from lemma 2 that $I^{s-h}=\omega^{s-h}$. Thus $I^{-h} \sigma \in \omega^{s-h}$ when $s-h \neq 0$ which shows that if $0<s-h<1$ theorem $A(0, \infty)$ reduces to $A\{q\}$ with $q=s-h$ and with $\sigma$ replaced by $I^{-h} \sigma$. When $s-h=0$ it is required in $A(0, \infty)$ that $I^{-s} \sigma \in \omega^{0}$ which is the condition of theorem $A\{0\}$ if $\sigma$ in this theorem is replaced by $I^{-h} \sigma=I^{-s} \sigma$. Thus $A(0, \infty)$ is proved when $0 \leqslant s-h<1$.

To prove the theorem when $s-h<0$ we use the identity

$$
(\lambda+t)^{-1}=\sum_{j=0}^{k-1}(-\lambda)^{j} t^{-j-1}+(-t)^{-k} \lambda^{k}(\lambda+t)^{-1}
$$

with $h-s \leqslant k<h-s+1$. Thus the left hand side of (3) equals a polynomial in $t^{-1}$ without constant term plus

$$
(-t)^{-k} \int_{0}^{\infty} \lambda^{k-h}(\lambda+t)^{-1} d \sigma(\lambda)
$$

Since $\sigma \in I^{s}$ it follows that $I^{-h+j} \sigma \in I^{s-h+j} \subset I^{s-h+k-1}=\omega^{s-h+k-1} \subset \omega^{0}$ which shows the convergence of the occurring integrals. In (5) $0 \leqslant s-(h-k)<1$ and the integral can be treated in the same way as the left hand side of (3) when $0 \leqslant s-h<1$. This completes the proof. 


\section{Proof of the unilateral Tauberian theorem}

When $0 \leqslant s-h<1$ the Tauberian theorem $T(0, \infty)$ is reduced to $T\{q\}$ and $T\{0\}$. The assumption $\sigma \in T^{s}$ gives $I^{-h} \sigma \in T^{s-h}$ according to lemma 6 . Hence if $0<s-h<1$ it follows from $T\{q\}, q=s-h$, that $I^{-h} \sigma \in \omega^{s-h}=I^{s-h}$. Lemma 3 shows that $\sigma \in I^{s}$. When $s-h=0$ the theorem $T\{0\}$ gives $I^{-h} \sigma \in \omega^{0}$ or $I^{-s} \sigma \in \omega^{0}$.

If $s \sim h<0$, the basic relation (4) implies that

$$
\int_{0}^{\infty}(\lambda+t)^{-1} d I^{-h} \sigma=a_{1} t^{-1}+o\left(t^{-1}\right), \quad t \rightarrow+\infty .
$$

Since $I^{-h} \sigma \in T^{s-h}$ and $s-h<0$ it follows from lemma 9 that $I^{-h} \sigma \in T^{0}$. Thus $T\{0\}$ shows that $I^{-h} \sigma \in \omega^{0}$. Because of this and theorem 1 formula (4) can be written

$$
\int_{0}^{\infty} \lambda^{-h} d \sigma-\int_{0}^{\infty} \lambda^{1-h}(\lambda+t)^{-1} d \sigma=a_{1}+a_{2} t^{-1}+\ldots+o\left(t^{s-h}\right)
$$

Letting $t$ tend to infinity one finds because of the second part of theorem 1 that

$$
\int_{0}^{\infty} \lambda^{-h} d \sigma=a_{1}
$$

and the formula reduces to

$$
\int_{0}^{\infty} \lambda^{1-h}(\lambda+t)^{-1} d \sigma(\lambda)=-a_{2} t^{-1}-\ldots+o\left(t^{s-h}\right)
$$

Thus, provided $s-h<0$, formula (4) can be replaced by a similar one with $h-1$ instead of $h$. If $s-(h-1)<0$ the procedure is repeated. Finally a formula is obtained in which $h$ is replaced by $h-r$ with $0 \leqslant s-(h-r)<1$. We are then in the case already considered and the proof is, accomplished.

\section{Inhomogeneous theorems}

Let $\chi=K \lambda^{s}$ when $s \neq 0$ and $\lambda \geqslant a>0$ and let $\chi=K \log \lambda$ when $s=0$ and $\lambda \geqslant a>0$. Here $K$ and $a$ are constants. For $0 \leqslant \lambda<a$ the function $\chi$ is arbitrarily defined so as to cause no trouble about the convergence at the origin of the integral

$$
\int_{0}^{\infty} \lambda^{-n}(\lambda+t)^{-1} d \chi(\lambda)
$$

It is easy to see that when $s<h+1$ the condition $I^{-h-1} \chi \in \omega^{0}$ is fulfilled which guarantees the convergence at infinity of the integral. Also $\chi \in T^{s}$.

If $\sigma$ is replaced by $\varphi-\chi$ in $A(0, \infty), T(0, \infty)$ these theorems are transferred into "inhomogeneous" theorems connecting the conditions $\varphi-\chi \in I^{s}$ or $I^{-s}(\varphi-\chi) \in \omega^{0}$ (when $s-h=$ integer) on one side and 
ARKIV FÖR MATEMATIK. Bd $4 \mathrm{nr} 45$

$$
\int_{0}^{\infty} \lambda^{-h}(\lambda+t)^{-1} d \varphi=\int_{0}^{\infty} \lambda^{-h}(\lambda+t)^{-1} d \chi+t^{-1} p\left(t^{-1}\right)+o\left(|t|^{s-h-1}\right)
$$

on the other. In the inhomogeneous Tauberian theorem the condition $\varphi \in T^{s}$ takes the place of $\sigma \in T^{s}$.

Simple calculations show that in these inhomogeneous theorems the integral (6) can be replaced by

$$
\begin{aligned}
& K \frac{\pi s}{\sin \pi(s-h)} t^{s-h-1} \text { when } s \neq 0, s-h \neq \text { integer, } \\
& K s(-1)^{s-h} t^{s-h-1} \log t \text { when } s \neq 0, s-h=\text { integer, } \\
& K \frac{\pi}{\sin (-\pi h)} \text { when } s=0, s-h \neq \text { integer, } \\
& K(-1)^{-h} t^{-h-1} \log t \text { when } s=0, s-h=\text { integer. }
\end{aligned}
$$

\section{A bilateral Abelian theorem}

In the bilateral case functions $\sigma(\lambda)$ are considered which are defined on $-\infty<\lambda<+\infty$ and of bounded variation over every finite subinterval. It is assumed that

$$
\int|\lambda|^{-n}|d \sigma(\lambda)|
$$

converges over a two-sided neighbourhood of the origin.

We are concerned with the study of the integral

$$
\int_{-\infty}^{+\infty}|\lambda|^{-h}(\lambda+t)^{-1} d \sigma(\lambda)
$$

which is supposed to converge. This means that $I^{-h-1} \sigma(\lambda)$ and $I^{-h-1} \sigma(-\lambda)$ are in $\omega^{0}$ when considered for positive values of $\lambda$. As in the unilateral case it is natural to assume $s \leqslant h+1$ and the case when $s=h+1$ is trivial. We therefore suppose $s<h+1$.

The part of (7) which comes from $-\infty<\lambda<0$ is transformed into an integral from 0 to $+\infty$ by the substitution $\lambda=-\mu$. Afterwards $\mu$ is replaced by $\lambda$. With

the result reads

$$
\begin{aligned}
& S(\lambda)=\sigma(\lambda)+\sigma(-\lambda), \\
& A(\lambda)=\sigma(\lambda)-\sigma(-\lambda),
\end{aligned}
$$

$$
\int_{0}^{\infty} \lambda^{1-h}\left(\lambda^{2}-t^{2}\right)^{-1} d S(\lambda)-t \int_{0}^{\infty} \lambda^{-h}\left(\lambda^{2}-t^{2}\right)^{-1} d A(\lambda)
$$




\section{ÅKe PLeIJEL, $A$ bilateral Tauberian theorem}

From this it follows that a relation

$$
\int_{-\infty}^{+\infty}|\lambda|^{-h}(\lambda+t)^{-1} d \sigma(\lambda)=t^{-1} p\left(t^{-1}\right)+o\left(|t|^{s-h-1}\right),
$$

holding when $t \rightarrow \infty$ along two opposite non real half rays, can be split into formulas

$$
\begin{array}{r}
\int_{0}^{\infty} \Lambda^{-h / 2}(\Lambda+T)^{-1} d A(\sqrt{\Lambda})=T^{-1} P_{1}\left(T^{-1}\right)+o\left(|T|^{s / 2-h / 2-1}\right) \\
\int_{0}^{\infty} \Lambda^{-\frac{n-1}{2}}(\Lambda+T)^{-1} d S(\sqrt{\Lambda})=T^{-1} P_{2}\left(T^{-1}\right)+o\left(|T|^{\frac{S}{2}-\frac{h-1}{2}-1}\right)
\end{array}
$$

valid when $T \rightarrow \infty$ along a half ray different from the negative real axis. Here $T=-t^{2}, \Lambda=\lambda^{2}$ and the polynomials $P_{1}, P_{2}$ are determined by $p(x)+p(-x)=$ $2 P_{1}\left(-x^{2}\right), p(x)-p(-x)=-2 x P_{2}\left(-x^{2}\right)$ when the polynomial $p$ is known. Evidently $\frac{1}{2} s<\frac{1}{2} h+1, \frac{1}{2} s<\frac{1}{2}(h-1)+1$ since $s<h+1$.

The bilateral Abelian theorem is now an immediate consequence of $A(0, \infty)$ applied to $(8),(9)$.

Theorem $A(-\infty,+\infty)$. If $s<h+1$ and $s-h$ is not an integer it follows from $\sigma(\lambda) \in I^{s}, \sigma(-\lambda) \in I^{s}$ that

$$
\int_{-\infty}^{+\infty}|\lambda|^{-h}(\lambda+t)^{-1} d \sigma(\lambda)=t^{-1} p\left(t^{-1}\right)+o\left(|t|^{s-h-1}\right)
$$

when $t \rightarrow \infty$ along non real half rays from the origin. Here $p$ is a polynomial. If $s-h$ is an integer the same result is valid under the supplementary conditions $I^{-s}(\sigma(\lambda)+\sigma(-\lambda)) \in \omega^{0}$ when $s-h$ is odd, $I^{-s}(\sigma(\lambda)-\sigma(-\lambda)) \in \omega^{0}$ when $s-h$ is even.

\section{The bilateral Tauberian theorem}

Theorem $T(-\infty,+\infty)$. Let $s<h+1$ and assume that $\sigma(\lambda) \in T^{s}, \sigma(-\lambda) \in T^{s}$ for positive values of $\lambda$. If with a polynomial $p$

$$
\int_{-\infty}^{+\infty}|\lambda|^{-n}(\lambda+t)^{-1} d \sigma(\lambda)=t^{-1} p\left(t^{-1}\right)+o\left(|t|^{s-h-1}\right)
$$

as $t \rightarrow \infty$ along non real half rays from the origin, then $\sigma(\lambda) \in I^{s}$ and $\sigma(-\lambda) \in I^{s}$ for $\lambda \rightarrow+\infty$.

Observe that the couple $A(-\infty,+\infty), T(-\infty,+\infty)$ does not show the same symmetric reciprocity as $A(0, \infty), T(0, \infty)$.

If we wanted to include also the case when $s=h+1$ we would conclude from the mere existence of the integral in $(10)$ that $I^{-s} \sigma(\lambda) \in \omega^{0}, I^{-s} \sigma(-\lambda) \in \omega^{0}$ which implies $\sigma(\lambda) \in I^{s}, \sigma(-\lambda) \in I^{s}$. 
But for certain exceptional cases occurring only when $s-h$ is an integer the proof of $T(-\infty,+\infty)$ is obtained by replacing $t$ in (10) by it, the new $t$ being real. If the resulting relation is split as in section 7 we obtain (8), (9) with $T$ real positive.

According to lemma 7 and 8 either $S(\sqrt{\Lambda})$ or $A(\sqrt{\Lambda})$ belongs to $T^{s / 2}$. Let us first assume $S(\sqrt{\Lambda}) \in T^{s / 2}$. Then $T(0, \infty)$ can be applied to $(9)$ with the result $S(\sqrt{\Lambda}) \in I^{s / 2}$. If $s-h$ is an odd integer $T(0, \infty)$ gives in addition the convergence of

$$
\int_{0}^{\infty} \lambda^{-s} d(\sigma(\lambda)+\sigma(-\lambda))
$$

which, however, is of no use in the rest of the proof. Provided $s-h$ is not an even integer theorem $A(0, \infty)$ yields

$$
\int_{0}^{\infty} \Lambda^{-\frac{h}{2}}(\Lambda+T)^{-1} d S(\sqrt{\Lambda})=T^{-1} P_{2}^{\prime}\left(T^{-1}\right)+o\left(|T|^{\frac{s}{2}-\frac{h}{2}-1}\right)
$$

where $P_{2}^{\prime}$ is a new polynomial. Addition and subtraction of (11), (8) lead to separate formulas for $\sigma(\sqrt{\Lambda})$ and $\sigma(-\sqrt{\Lambda})$. Application of $T(0, \infty)$ to these formulas finally shows that $\sigma(\sqrt{\Lambda}), \sigma(-\sqrt{\Lambda}) \in I^{s / 2}$ and the conclusion of $T(-\infty$, $+\infty)$ follows from lemma 5 .

If instead $A(\sqrt{\Lambda}) \in T^{s / 2}$ the same method is applied but with starting point in (8). The same result is obtained provided $s-h$ is not an odd integer. If $s-h$ is an even integer we find the additional result that

$$
\int_{0}^{\infty} \lambda^{-s} d(\sigma(\lambda)-\sigma(-\lambda))
$$

exists.

The exceptional cases $s-h=$ even integer, $S(\lambda) \in T^{s}$ and $s-h=$ odd integer, $A(\lambda) \in T^{s}$ remain to be considered.

\section{Proof of the bilateral Tauberian theorem in the exceptional cases}

To take care also of these cases we must use (10) not only along one couple of opposite half rays but along two. Since it is not more complicated to consider $n$ couples we do this.

The point of departure is the partition in partial fractions

$$
2 n t^{2 n-\alpha-1} \lambda^{\alpha}\left(\lambda^{2 n}+t^{2 n}\right)^{-1}=-\sum_{k=0}^{2 n-1} \varepsilon_{k}^{\alpha+1}\left(\lambda-\varepsilon_{k} t\right)^{-1}
$$

where $\alpha=0,1,2, \ldots(2 n-1)$ and

$$
\varepsilon_{k}=\exp \left(\frac{\pi i}{2 n}+k \frac{\pi i}{n}\right)
$$




\section{ÅKe PLEIJEL, $A$ bilateral Tauberian theorem}

In (10) $t$ is now replaced by $-\varepsilon_{k} t, k=0,1,2, \ldots(2 n-1)$, after which the resulting formulas are multiplied by $\varepsilon_{k}^{\alpha+1}$ and added. The result is condensed by help of (12) and occurring integrals from $-\infty$ to 0 are transformed into integrals from 0 to $+\infty$. At last the new variables $\Lambda=\lambda^{2 n}, T=t^{2 n}$ are introduced.

The formulas deduced in this way are

$$
\int_{0}^{\infty} \Lambda^{-\frac{h-\alpha}{2 n}}(\Lambda+T)^{-1} d\left(\sigma\left(\Lambda^{\frac{1}{2 n}}\right)-(-1)^{\alpha} \sigma\left(-\Lambda^{\frac{1}{2 n}}\right)\right)=T^{-1} P_{\alpha}\left(T^{-1}\right)+o\left(|T|^{\frac{s}{2 n}-\frac{h-\alpha}{2 n}-1}\right)
$$

$\alpha=0,1,2, \ldots(2 n-1)$. They are certainly valid when $T \rightarrow \infty$ along the positive real axis.

According to lemma 8 and 7 we know that

$$
\sigma\left(\Lambda^{\frac{1}{2 \bar{n}}}\right)-(-1)^{\alpha} \sigma\left(-\Lambda^{\frac{1}{2 n}}\right) \in T^{\frac{s}{2 n}}
$$

holds either for $\alpha$ odd or even. Assume first that it holds for $\alpha$ odd. Theorem $T(0, \infty)$ can then be applied to any of the formulas (13) with $\alpha$ odd. The result is

$$
\sigma\left(\Lambda^{\frac{1}{2 n}}\right)+\sigma\left(-\Lambda^{\frac{1}{2 n}}\right) \in I^{\frac{s}{2 n}}
$$

Next chose $\alpha$ even and such that

$$
\frac{s}{2 n}-\frac{h-\alpha}{2 n}
$$

is not an integer. This is possible if only $n>1$ i.e. if there is more than one even $\alpha, 0 \leqslant \alpha<2 n$. With the chosen even $\alpha$ theorem $A(0, \infty)$ shows because of (15) that

$$
\int_{0}^{\infty} \Lambda^{-\frac{h-\alpha}{2 n}}(\Lambda+T)^{-1} d\left(\sigma\left(\Lambda^{\frac{1}{2 n}}\right)+\sigma\left(-\Lambda^{\frac{1}{2 n}}\right)\right)=T^{-1} P_{\alpha}^{\prime}\left(T^{-1}\right)+o\left(|T|^{\frac{s}{2 n}-\frac{h-\alpha}{2 n}-1}\right),
$$

where $P_{\alpha}^{\prime}$ is a polynomial. This relation and formula (13) with the same even $\alpha$ lead to relations for

$$
\int_{0}^{\infty} \Lambda^{-\frac{h-\alpha}{2 n}}(\Lambda+T)^{-1} d \sigma\left( \pm \Lambda^{\frac{1}{2 n}}\right)
$$

from which it follows by help of $T(0, \infty)$ that

$$
\sigma\left( \pm \Lambda^{\frac{1}{2 n}}\right) \in I^{\frac{s}{2 n}}
$$

According to lemma 5 this is equivalent to

$$
\sigma(\lambda) \in I^{s}, \quad \sigma(-\lambda) \in I^{s} .
$$

The case when (14) is valid with $\alpha$ even is similarly treated with the same result. 


\section{Inhomogeneous form of the bilateral Tauberian theorem}

With

when $s \neq 0$ and

$$
\chi(\lambda)= \begin{cases}K_{1} \lambda^{s} & \text { for } 0<a \leqslant \lambda \\ K_{2}(-\lambda)^{s} & \text { for } \lambda \leqslant-a\end{cases}
$$

$$
\chi(\lambda)= \begin{cases}K_{1} \log \lambda & \text { for } 0<a \leqslant \lambda \\ K_{2} \log (-\lambda) & \text { for } \lambda \leqslant-a\end{cases}
$$

when $s=0$ the theorem $T(-\infty,+\infty)$ takes the inhomogeneous form

Theorem $T(-\infty,+\infty)$. If $s<h+1$ and $\varphi(\lambda) \in T^{s}, \varphi(-\lambda) \in T^{s}$ and if with a polynomial $p$

$$
\int_{-\infty}^{+\infty}|\lambda|^{-h}(\lambda+t)^{-1} d \varphi(\lambda)=\int_{-\infty}^{+\infty}|\lambda|^{-h}(\lambda+t)^{-1} d \chi(\lambda)+t^{-1} p\left(t^{-1}\right)+o\left(|t|^{s-h-1}\right)
$$

as $t \rightarrow \infty$ along non real half rays from the origin, then $\varphi(\lambda)-\chi(\lambda) \in I^{s}, \varphi(-\lambda)-$ $\chi(-\lambda) \in I^{s}$ for $\lambda \rightarrow+\infty$.

In this theorem the integral on the right hand side

$$
\int_{0}^{\infty} \lambda^{-h}(\lambda+t)^{-1} d \chi(\lambda)+\int_{0}^{\infty} \lambda^{-h}(\lambda-t)^{-1} d \chi(-\lambda)
$$

can be replaced by expressions directly obtained from section 6 . The expression for the first integral in (16) shall be real when $t$ is real positive (we assume $K_{1}$ and $K_{2}$ real) and the one replacing the second integral shall be real when $t$ is real negative.

\section{RE FEREN CES}

1. Hardy G. H. and Litruewood J. E., Notes on the theory of series (XI): On Tauberian theorems, Proceedings of the London Mathematical Society, Series 2, Vol. 30, Part 1 (1930), 23-37.

2. Pleidel A., Certain indefinite differential eigenvalue problems - The asymptotic distribution of their eigenfunctions, Partial Differential Equations and Continuum Mechanics, Madison, The University of Wisconsin Press (1961), 19-37. 